Nueva Revista de Filología Hispánica (NRFH), LXVIII, 2020, núm. 2, 747-766

ISSN 0185-0121; e-ISSN 2448-6558; DOI: 10.24201/nrfh.v68i2.3656

\title{
NOTAS ETIMOLÓGICAS SOBRE LAS VARIANTES DE ENJUNDIA Y ESPONJA EN IBERORROMANCE
}

\author{
ETYMOLOGICAL NOTES ON THE VARIANTS \\ OF ENJUNDIA AND ESPONJA IN IBERO-ROMANCE
}

\author{
CÉSAR Gutiérrez \\ Wake Forest University \\ gutiermc@wfu.edu
}

\begin{abstract}
RESUMEN: En este artículo se estudian las variantes diacrónicas y diatópicas de enjundia y esponja en iberorromance para determinar cuáles son los étimos de cada una de ellas en esta área de la Romania. A partir del análisis fonético de los posibles étimos (AXUNGIAM y *AXUNGUlam para enjundia y sus variantes; SPONGIAM y *SPONGULAM para esponjay sus variantes), se concluirá que aquellos con la secuencia-NGIV-son el origen de las variantes con $[\mathrm{n}],[\mathrm{nts}],[\mathrm{ndz}](>[\mathrm{n} \theta]),[\mathrm{n}(\mathrm{t}) \mathrm{f}],\left[\mathrm{n}(\mathrm{d})_{3}\right]\left(>\left[\tilde{\mathrm{V}}_{3}\right],[\mathrm{nx}]\right)$ $\mathrm{y}[\mathrm{nd}]$, y que aquellos otros con -NGUL- lo son de las variantes que han evolucionado a $[\mathrm{n} K]$ y $[\kappa]$.
\end{abstract}

Palabras clave: enjundia; esponja; etimología; historia del léxico iberorromance; cambio fónico.

Aвstract: In this article, the historical and geographical variants of enjundia and esponja in Ibero-Romance are studied to determine the etymons of each of those variants in this Romance area. Through the phonetic analysis of the potential etymons (AXUNGIAM and *AXUNGULAM for enjundia and its variants; SPONGIAM and *SPONGULAM for esponja and its variants), it will be concluded that those containing the sequence -NGIV- are the source for the variants exhibiting [n], [nts], [ndz] (> $[\mathrm{n} \theta]),\left[\mathrm{n}(\mathrm{t}) \int\right],\left[\mathrm{n}(\mathrm{d})_{3}\right]\left(>\left[\tilde{\mathrm{V}}_{3}\right],[\mathrm{nx}]\right)$ and $[\mathrm{nd}]$, while those containing the sequence -NGUL- are the source for the variants exhibiting $[\mathrm{n} K]$ and $[K]$.

Keywords: enjundia; esponja; etymology; history of the Ibero-Romance lexicon; sound change.

Recepción: 22 de noviembre de 2018; aceptación: 27 de marzo de 2019.

D.R. (C) 2020. Nueva Revista de Filología Hispánica Licencia Creative Commons Attribution-NonCommercial (CC BY-NC) 4.0 International 


\section{INTRODUCCIÓN}

A pesar de las ostensibles diferencias formales y semánticas entre sí, las variantes iberorrománicas de enjundia y esponja tienen orígenes y evoluciones fonéticas bastante parejos. La etimología de estas voces ha venido siendo tenazmente discutida desde los primeros estudios sobre el tema: para enjundia y sus variantes se han planteado AXUNGIAM y *AXUNGULAM, y para esponja y las suyas, SPONGIAM, *SPONGAM y*sPONGULAM (véanse infra sus apartados correspondientes). Ante esta multiplicidad de propuestas, el objetivo del presente trabajo es intentar esclarecer la procedencia de todas estas variantes en iberorromance por medio del análisis fonético de sus potenciales étimos. Concretamente, se prestará atención a las secuencias latinas -NGIV-y -NGUL- (fonéticamente [ygjV] y [ygul]) para delimitar con el mayor grado de fiabilidad qué desarrollos fueron viables a partir de ellas y cuáles fueron, por el contrario, poco factibles (véase "Análisis fonético”). Las conclusiones de este análisis permitirán sugerir la hipótesis de que, salvo las formas gallegas y portuguesas con $[\mathrm{n} \kappa]$ y $[K]$, que están emparentadas con *AXuNGULAM y *SPONGULAM, las demás variantes iberorrománicas, que exhiben $[\mathrm{n}],[\mathrm{nts}],[\mathrm{ndz}](>[\mathrm{n} \theta])$, $\left[\mathrm{n}(\mathrm{t}) \int\right],\left[\mathrm{n}(\mathrm{d})_{3}\right]\left(>\left[\tilde{\mathrm{V}}_{3}\right],[\mathrm{yx}]\right)$ y [nd], provienen probablemente de AXUNGIAM y SPONGIAM. Finalmente, se evaluará el papel de los diminutivos AXUNGIOLA o SPONGIOLA dentro de esta explicación fonética (véase "Análisis morfológico").

\section{ENJUNDIA}

Corominas (DCECH, s.v. enjundia) señala que esta voz en castellano proviene de AXUNGIAM 'grasa', opinión que comparte, con alguna reserva, García de Diego (DEEH, s.vv. axungiay * axungula), quien en su diccionario la encuadra tanto entre los descendientes de AXUNGIAM como entre los de *AXUNGULAM. Es al considerar el conjunto de las variantes iberorrománicas cuando las posturas de ambos autores empiezan a distanciarse.

Atendiendo a cómo haya evolucionado la parte final de la palabra, las variantes de enjundia que encontramos en iberorromance se pueden agrupar en cuatro categorías, tal como se muestra en (1).

(1) Variantes de enjundia en iberorromance ${ }^{1}$ : a. [n]: cat. ensunya (DCVB, s.v. ensunya).

${ }^{1}$ Lista de abreviaturas referidas a las lenguas y dialectos mencionados a lo largo del artículo: ant. $=$ antiguo; arag. $=$ aragonés $;$ ast. $=$ asturiano $;$ avul. $=$ avulense $;$ burg . = burgalés; cast. $=$ castellano $;$ cat. $=$ catalán $;$ extrem.$=$ extremeño $;$ fr. $=$ francés; gall . $=$ gallego; gr. $=$ griego; leon.$=$ leonés; occ.$=$ occitano; palen.$=$ palentino; port. $=$ 
b. $[\mathrm{n} K],[K],[\mathrm{nlj}]$ : port. ant. enxunlha; port. anxulha, enxulha, inxulia ${ }^{2}$; gall. enxunlla, enxulla (cf. DEEH, s.v. *axungula); gall. ensulla 'enjundia', 'unto', 'grasa de las aves' (cf. TLPGP).

c. [n $\theta$ ]: sant. juncia 'unto de la gallina' (García Lomas 1966) y

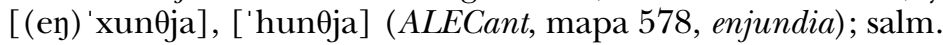
y extrem. ['hun $\theta$ ja] (Espinosa, 1935, § 20); palen., burg., avul. y segov. [(en)'xun $\theta j a]$ (ALCyL, mapa 566, enjundia) ${ }^{3}$.

d. [nd]: cast. enjundia; port. enxúndia; leon. enxundria, enxundriga (Casado Lobato, 1948, § 41), [(in)'sundja] (ALCyL, mapa 566, enjundia); salm. [ay'xundja], ['hundja] (Espinosa 1935, § 20); arag. anxundia (cf. DEEH, s.v. * axungula), ensundia (Huesca) e injundia (Caspe) (cf. Andolz 1991, s.v. enjundia) ${ }^{4}$.

Corominas piensa que todas estas variantes tienen su origen en AXUNGIAM: en ( $1 a)$-NGIV- sigue la palatalización de las secuencias -NGE,I- (fonéticamente [yge,i]) común en catalán (cf. CINGERE > cat. cenyir [so'ni]; IUNGERE > cat. junyir [3u'ni]; GINGIVAM > cat. genyiva [3ə'nißə]; véanse $D C V B$ y Moll 1952, § 162) y en castellano (cf. CINGERE > cast. ceñir; IUNGERE > cast. uñir; TINGERE > cast. teñir; véase Lloyd 1993, p. 409); en (1b) [ $K$ ], presente en gallego y en portugués, es derivada por Corominas linealmente de las variantes con [n], alegando una disimilación entre las nasales [n] y [n] (*enxunya $>$ ensulha) (DCECH, s.v. chuleta); en (1c), por su parte, [n $\theta]$ lo trae del estadio con la africada [ndz] al que llegó -NGIV-junto con la -NGE,Ide determinadas palabras latinas en castellano (cf. GINGIVAM $>$ cast. enzia; IUNGERE > cast. unzir; *RINGELLAM > cast. renzilla; *SINGELLUM > cast. senzillo; véanse Lloyd 1993, p. 410, y Menéndez Pidal 1941, § $47_{2 b}$ ); por fin, en ( $\left.1 d\right)$ [nd] también proviene a juicio de Corominas, que sigue a Cornu (1906, p. 991) en este punto, de -NGIV-, pero con una disimilación en *[en'Jundza] entre [ $]$ ] y el fragmento fricativo de $[\mathrm{dz}]$ : esta disimilación acarreó la elisión de [z] y condujo a [nd] (DCECH, s.v. enjundia $)^{5}$.

portugués; ribag. $=$ ribagorzano; salm. = salmantino; sant. = santanderino; segov. = segoviano.

${ }^{2}$ Esta forma se registra en la villa de Arcos de Valdevez, distrito de Viana do Castelo (Alves Pereira 1933). Todo apunta a que se trata de una despalatalización de $[K]$ motivada por la descomposición gestual de esta consonante $([K]>[\mathrm{lj}])$. Un proceso similar se observa en otras palabras de este dialecto recogidas en el mismo trabajo por el propio Alves Pereira: coleijo (port. colegio), frainja (port. franja), queinlha (port. quelha $<$ CANALICULAM).

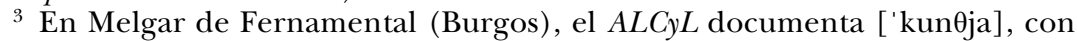
presumible reforzamiento de la $[\mathrm{x}]$ inicial en $[\mathrm{k}]$.

${ }^{4}$ Y en varios países de América, infundia 'enjundia o gordura de un ave, especialmente de la gallina' (DA, s.v. infundia).

${ }^{5}$ Michaëlis de Vasconcellos (1910), hablando sobre el étimo de esponlha y espunlha en portugués medieval, que ella deriva de sPongulam, trae enxunlha, enxulha, enxunha, enxundia y enxunda de AXUNGIAM. 
A esta descripción de los hechos, García de Diego objeta que las formas gallegas y portuguesas con $[K]$ no pueden estar ligadas a AxUNGIAM por medio de la confusión de $[\mathrm{n}]$ con $[\kappa]$, sino que, como prueban el portugués antiguo enxunlha y el gallego moderno enxunlla, tienen que proceder de *AXUNGULAM (cf. SINGULARIUM > port. ant. Senlheiro; ungulam > gall. unlla). Asimismo, observa que las variantes con [nd] como enxundia pueden venir de *AXUNGULAM (cf. SINGULOS > cast. sendos), pero también de AXUngiam, vista la evolución de -NGE,I- a [ndz] en castellano. Con esto, García de Diego da a entender que acepta implícitamente los argumentos de Cornu y de Corominas sobre la disimilación de las sibilantes $\left[\int\right]$ y [z] en * [en'fundza]

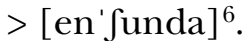

(2) Étimo de enjundia y de sus variantes en iberorromance según Corominas (DCECH, s.vv. chuleta y enjundia): AXUNGIAM [ngj]

$>[\mathrm{n}]>[\Lambda]$ : cat. ant. ensunya; port. enxulha, enxunlha.

$>[\mathrm{nts}],[\mathrm{ndz}]>[\mathrm{n} \theta]$ : extrem. juncia.

$>\left[\int \ldots \mathrm{ndz}\right]>\left[\int \ldots \mathrm{nd}\right]$ : cast. enjundia; port. exunda, enxundia.

(3) Étimos de enjundia y de sus variantes en iberorromance según García de Diego (DEEH, s.vv. axungia y * axungula):

$a$. AXUNGIAM [ygj]

$>$ [n]: cat. ant. enxunya; cat. ansunya.

$>$ [nd]: cast. enjundia.

b. *aXungulam [ygul]

$>[\mathrm{n} K]>[K]>[\mathrm{lj}]$ : gall. enxunlla, enxulla; port. ant. enxunlha; port. enxulha; port. inxulia.

$>$ [n $\theta]$ : extrem. y sant. juncia.

$>$ [nd]: cast. enjundia; extrem. jundia; port. enxunda; arag. anxundia.

En estas discrepancias, lo único que parece indiscutible es que el catalán ensunya se deriva de AXUNGiam, no de *AXUNGUlam, ya que en esta lengua -NGUL- da [ygl], sin palatalización del grupo [gl] (cf. SINGULAREM > senglar [sən'gła]; UNGULAM > ungla ['ungłə]; véanse Badía Margarit 1951, $\S 98_{1}$, y Moll 1952, $\left.§ 174\right)$. Sin embargo, para las demás variantes peninsulares necesita elucidarse si todas ellas provienen exclusivamente de AXUNGIAM -como plantean Michaëlis de Vasconcellos, Meyer-Lübke ( $R E W$, s.v. 846 axungia) y Corominas- o si algunas vienen de AXUNGIAM y otras de *AXUNGULAM -como propone García de Diego.

${ }^{6}$ Esta explicación de Corominas ya está íntegra en el DCELC, publicado entre 1955 y 1957 (el $D C E C H$ sólo añade una referencia bibliográfica a un trabajo de Jud), por lo que García de Diego la conocía cuando redactó los artículos de axungia y *axungula de la $2^{\text {a }}$ edición del $D E E H$ antes de su fallecimiento en 1978. 
ESPONJA

Las variantes de esponja en iberorromance se pueden encuadrar en una de las siguientes seis categorías:

(4) Variantes de esponja en iberorromance:

a. [n]: arag. espiña (REW, s.vv. 8173 spongia, *sponga), espuña 'piedra esponjosa' (DEEH, s.v. spongia).

b. $[\mathrm{n} K],[K]$ : port. ant. espunlha y gall. espunlla 'tumor de los caballos'; gall. espulla 'verruga fungosa de los árboles' (cf. DEEH, s.v. *spongula); port. espulha y pulha 'verruga' (Viana do Castelo) (cf. Lourenço Loução 1931); gall. pulla 'verrugas que les salen a las vacas en la lengua' (Porto do Son), espunllas 'verruga grande con grietas' (Lage) (cf. TLPGP).

c. [nts], [ndz], [n $]$ ]: cast. esponça (finales del siglo Xıı; cf. Castro 1921); cast. esponza (1474; cf. Tallgren 1907); sant. espuncia y escuncia 'verrugas del ganado vacuno joven situadas alrededor de los ojos o narices' (cf. García Lomas 1966); ast. escuncia 'verruga que aparece en la ubre' (cf. Neira Martínez y Piñeiro 1989); leon. espuncia 'padrastro' (DEEH, s.v. *spongula); extrem.

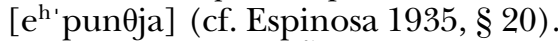

d. $\left[\mathrm{n}(\mathrm{t}) \int\right],\left[\mathrm{n}(\mathrm{d})_{3}\right],\left[\tilde{\mathrm{V}}_{3}\right]$ : gall. esponxa [es'ponfa] (DPLG, s.v. esponxa); port. esponja [iٔ' põza]; cat. esponja (barcelonés y balear [əs' ponzə], tortosano y castellonés [as'pondza], valenciano [es' pontfa]) (cf. DCVB, s.v. esponja); arag. esponchá 'embeber un cuerpo sólido algún líquido’ (Benasque) (cf. Andolz 1991, s.v. esponchá).

e. [ [ $\mathrm{yx}]$ : cast. esponja; arag. esponja 'parte del vientre del animal donde apoya el braguero' (cf. Andolz 1991, s.v. esponja).

f. [nd]: cast. espundia 'tumor de los caballos' (DEEH, s.v. *spongula $^{7}$; gall. espundia 'tumoraciones que le salen a las vacas en el vientre y la ubre' y 'verrugas' (Gudiña) (cf. TLPGP); extrem.

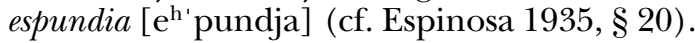

De nuevo es Corominas (DCECH, s.v. esponja) quien defiende un solo étimo para todos los derivados iberorromances. Para él, esponja en portugués, castellano y catalán es un descendiente semiculto y completamente autóctono en cada una de estas lenguas del latín SPONGIAM (del gr. $\sigma \pi 0 \gamma \gamma i \alpha ́)$. Corominas rechaza por tanto la creencia de Meyer-Lübke ( $R E W$, s.vv. 8173 spongia, *sponga) de que esponja sea un galicismo en los romances hispánicos adaptado del francés éponge. En la entrada correspondiente a espundia (DCECH, s.v. espundia), considera esta palabra otro caso de evolución semiculta a partir de SPONGIAM, y vuelve a insistir, por un lado, en la inexistencia de la forma reconstruida *sPONGAM, y por otro, en la certeza de que todas las

\footnotetext{
${ }^{7}$ Las ocurrencias más antiguas de esta forma en castellano aparecen en el Tesoro de la medicina (1431) y en el Libro de albeitería (1564) (cf. CORDE).
} 
variantes románicas, incluidas, por supuesto, las iberorromances, se derivan de spongiam. Como sucedía con enjundia, Corominas achaca la desfricativización de la africada en el estadio [es 'pondza] a una disimilación con la [s] precedente, y justifica las variantes gallegas y portuguesas con [nK] (port. ant. espunlha y gall. espunlla) mediante -NGIV- > $n y^{8}>-n l h-$, cuyo último paso estuvo inducido por el influjo que sobre esponya ejerció unlha, vocablo que en los textos portugueses suele aparecer en su vecindad.

Entre los partidarios de más de un étimo, Meyer-Lübke ( $R E W$, s.vv. 8173 spongia, *sponga) establece sPONGIAM como base para algunos romances como el italiano spugna, el friulano spondze o el francés antiguo espongne, así como también el aragonés espiña, el castellano espundia y el gallego y portugués antiguo espunlha; el reconstruido * SPONGa fue la base del veneciano sponga, del provenzal esponga y del francés éponge, que, importado al sur, dio esponja en catalán, castellano y portugués. Meyer-Lübke muestra de este modo su desacuerdo con Michaëlis de Vasconcellos (1910), quien, aunque igualmente era favorable a dos étimos, había conjeturado SPONGIAM para el portugués esponjay el gallego esponxa, $\mathrm{y}$ *SPONGULAM, diminutivo de * SPONGAM, para el portugués espunlha ${ }^{9}$.

García de Diego (1943, §560; DEEH, s.v. spongia y *spongula) coincide con Michaëlis de Vasconcellos en los dos étimos propuestos, aunque aporta más ejemplos de otros iberorromances.

(5) Étimos de esponja y de sus variantes en iberorromance según García de Diego (1943, § 560; DEEH, s.vv. spongia y *spongula):

a. SPONGIAM [ygj]

$>$ [n]: arag. espuña 'piedra esponjosa'.

$>$ [nts], [ndz]: cast. ant. esponza.

$b$. *SPONGUlam [ngul]

$>[\mathrm{nx}]$ : cast. esponja.

$>[\mathrm{n} K]>[K]$ : port. ant. espunlha y gall. espunlla 'tumor de los caballos'; gall. espulla 'verruga fungosa de los árboles'; port. espulha 'verruga'.

$>$ [nӨ]: leon. espuncia 'padrastro'; extrem. espuncia 'úlcera'.

\footnotetext{
${ }^{8} \mathrm{Al}$ no ofrecerlo en transcripción fonética, es difícil saber si con este -ny-quiere representar [n] o [nz].

${ }^{9}$ Ese ejemplo de espunlha, junto con otro de esponlhas en el título del párrafo ("O Liij capitollo he das esponlhas que naçem aos cauallos") (véase la edición de Pereira, 1909), se encuentra en el tratado de albeitería del Mestre Giraldo, compuesto en Lisboa en 1318 y conservado en una copia del siglo xv. Es interesante que, como hace constar la propia Michaëlis de Vasconcellos, ese mismo espuntha sea glosado en una nota al margen con esponias en letra de mediados o finales del siglo XVI. Esto podría indicar la falta de familiaridad de algunos hablantes de portugués con la forma con $[\mathrm{n} K]$ ya para el Renacimiento.
} 
$>$ [nd]: cast. espundia 'tumor de los caballos'.

$>$ [nt $]$ : ribag. esponcha 'esponja' ${ }^{10}$.

En esta ocasión, García de Diego no hace ninguna advertencia acerca de que algunas de las variantes clasificadas por él bajo *SPONGULAM puedan venir de SPONGIAM, como sí decía de algunas de los de *AXungulam con respecto a AXungiam. Esto resulta muy llamativo dados los enormes paralelismos formales entre estas cuatro voces y sus derivados iberorromances. Resulta también sorprendente que este autor no incluya ningún ejemplo del catalán en su lista, sobre todo porque, en conformidad con las pronunciaciones modernas citadas en $(4 d)$, muchas de las variantes gráficas halladas en la documentación medieval y renacentista catalana son susceptibles de interpretarse como portadoras de fricativas o africadas alveopalatales -v.gr. esponge (finales del siglo XIII); sponja (siglo XIV); espòngea (segunda mitad del siglo xIV); sponga (1492); sponia (1491); y el topónimo Esponja; cf. DCVB, s.v. esponja, y DECat, s.v. esponja-; otras podrían leerse con nasal palatal -esponya (siglo xv) y los topónimos Espunya y Espunyola; cf. DCVB, s.v. esponja-; y otras aún, con [nd] -espòndia (1523); cf. DECat, s.v. esponja.

Al igual que con enjundia, puesto que la secuencia secundaria [ygl] no palataliza en catalán, parece que sólo las formas de esta lengua pueden vincularse, sin sombra de duda, a spongiam. Por lo demás, la división de opiniones entre los especialistas en lo tocante al origen de las variantes de esponja en iberorromance sigue dejando bastantes interrogantes sobre si todas ellas se remontan a un único étimo o si remiten a varios.

\section{ANÁLISIS FONÉTICO}

Con el fin de aclarar la filiación etimológica de enjundia y esponja, así como de sus respectivas variantes, a continuación se acomete el análisis fonético de las secuencias latinas -NGIV-y -NGUL-.

\section{La evolución de -NGIV-en iberorromance}

En los grupos [gj] intervocálicos del latín vulgar, la fusión gestual entre la [g] y la yod resultó en castellano en una obstruyente palatal sonora (v.gr. EXAGIUM > ensayo; FAGEAM > haya; FUGIO > huyo; PLAGEAM $>$ playa). Esta obstruyente ha sido descrita tradicionalmente

${ }^{10}$ Este ejemplo lo toma García de Diego de Ferraz y Castán (1934), quien no da esponcha 'esponja', sino el verbo esponchar con el significado de 'empapar' ("Ixes sopes se han esponchau prou"). 
como la fricativa [j] (Penny 2006, $§ 2.5 .2 .2$ ) o la aproximante $[\mathrm{j}]$ (Ariza 2012, pp. 165-169; Lloyd 1993, p. 397; Menéndez Pidal 1941, $\left.\S 53_{3}\right)^{11}$. No obstante, ninguna de estas propuestas resuelve satisfactoriamente la incógnita de cómo [dj, gj] latinovulgares pudieron dar lugar a /j/ y / ts, dz/ (>/ / /) en castellano sin confundirse con la /3/ salida de [lj] y [kl, gl] secundarias (v.gr. MULIEREM > mujer; OCULUM $>$ ojo; TEGULAM > teja).

Frente a estos planteamientos, otros más recientes catalogan el resultado de [gj] como la oclusiva alveopalatal [f]. Recasens (2011), retomando las observaciones de otros romanistas (MeyerLübke 1890, t. 1, § 403; Pope 1934, § 283 ss.), utiliza datos experimentales y comparativos para defender que las oclusivas (alveo)palatales $[\mathrm{c}, \mathrm{J}]$ fueron los antecedentes de las africadas tanto alveolares ([ts, $\mathrm{dz}]$ ) como alveopalatales $\left(\left[\mathrm{t} f, \mathrm{~d}_{3}\right]\right)$ en romance. Desde una perspectiva más dialectal y cronológica, Zampaulo (2013) aprovecha la oposición actual entre /j/ y /ł/ (cayó / ka'jo/; calló / ka'ło/) en el español argentino de Corrientes y en el de la Amazonia peruana como evidencia para explicar por qué la palatal procedente de [lj] y [kl, gl] secundarias $(/ \mathrm{j} /)$ no se fusionó en el castellano de orígenes con la procedente de $\left[\mathrm{j}, \mathrm{j}\right.$ : , dj, gj, ge, $\left.\mathrm{i},{ }^{\prime} \varepsilon\right](/ \mathrm{J} /)$, siendo posible que cada una de ellas siguiera evoluciones independientes.

Aceptando lo planteado por Recasens y Zampaulo, es presumible que en -NGIV- el grupo [gj] también desembocara en [f], y que así se formara $[\mathrm{nJ}]$. De esta nueva secuencia se pudo originar la [n] en catalán (ensunya) y en aragonés (espiña, espuña) por medio de un enmascaramiento acústico de la nasal sobre la oclusiva siguiente ([nJ] > $[\mathrm{n}(:)])$. Este proceso es idéntico al sufrido por otras secuencias de nasal + oclusiva sonora en romance, especialmente en los iberorromances orientales $-\mathrm{cf}$. [mb] $>$ [m(:)], LUMBUM $>$ cat. llom, UMBILICUM $>$ arag. melico; [nd] $>$ [n(:)], DEMANDĀRE $>$ cat. demanar, FUNDUM $>$ arag. ant. fonno, SPONDAM $>$ arag. ant. sponna; [yg] $>[\mathrm{y}(:)]$, SINGULOS $>$ ['singlos] > ['sin(:)los] > cast. sendos (Gutiérrez 2018; Badía Margarit 1951, § 80, Zamora Vicente 1967, pp. 236-237).

Con respecto a las africadas alveolares [nts] y [ndz] del astur-leonés (juncia y espuncia, escuncia) y del castellano (esponça, esponza), y a las africadas y fricativas alveopalatales $\left[\mathrm{n}(\mathrm{t}) \int\right]$ y $\left[\mathrm{n}(\mathrm{d})_{3}\right]$ del gallego $\left(>\left[\mathrm{n} \int\right]\right.$, esponxa), portugués, aragonés, catalán y castellano $(>[\mathrm{nx}]$, esponja), se debe tener en cuenta que el punto de articulación específico de una consonante alveopalatal surgida de una oclusiva velar puede ser bastante variable en una misma lengua: dependiendo de factores como el hablante, el contexto o la posición prosódica, su punto de articulación puede ubicarse en la región alveolar, en la alveopalatal, en la mediopalatal o en la postpalatal (Recasens y Espinosa,

${ }^{11}$ LAPESA $\left(1981, \S 18_{3}\right)$ : "Los grupos /d + yod/, /g + yod/ se redujeron a [j] o [y] (adjutare > ayutare)". 
2006 y 2009). Este grado de variación intralingüística puede ayudar a comprender las múltiples alternativas evolutivas que se derivan, en romance en general y en castellano en particular, de la $[\mathrm{f}]$ nacida tanto de -NGIV-como de -NGE,I-

Efectivamente, las secuencias -NGE,I- han llegado usualmente a dos soluciones en castellano: [n] (v.gr. CINGERE > ceñir, FINGERE $>$ feñir, TANGERE > tañer) o [nts], [ndz] -después [n $\theta]-\left({ }^{*}\right.$ SINGELLUM $>$ senziello, GINGIVAM > enzia; véanse Lloyd 1993, pp. 409-410, y Menéndez Pidal 1941, $\left.\S 47_{2 b}\right)$. A veces, un mismo étimo ha dado lugar a dobletes (FRANGERE > frañiry franzir, IUNGERE > uñiry unzir, RINGERE $>$ reñir y renzilla, de *RINGELLAM). En otros iberorromances como el asturleonés se ha producido el resultado [nd] para estas secuencias: FRANGERE $>$ ast. frander, frender; GINGIVAM > extrem. endiva (cf. DEEH). Y fuera de la Península Ibérica, otras lenguas románicas manifiestan asimismo esta doble evolución de -NGE,I-: en italorromance constan varios dobletes en donde uno de los miembros tiene [n] y el otro [nd3] (v.gr. FINGERE > fignere/ fingere; PLANGERE > piagnere/ piangere, PUNGERE > pugnere/ pungere, véase Rohlfs 1966, § 256); en la Galorromania la alternancia es entre [nd] y [n] (v.gr. FRANGERE $>$ fr. fraindre, occ. ant. franher, IUNGERE $>$ fr. joindre, occ. ant. jonher, PLANGERE $>$ fr. plaindre, occ. ant. planher; cf. FEW ${ }^{12}$.

Según esta gran variabilidad de las secuencias latinas -NGE,I- en su desarrollo, es asumible que las -NGIV-de AXUNGIAM y SPONGIAM pasaran primero a $\left[\mathrm{nJ}_{\mathrm{J}}\right.$ y que de este estadio se originaran en paralelo bien [nts], [ndz], o bien [n(t)f], [n(d)3]: [nts], [ndz] fue el desenlace para aquellos hablantes o dialectos en los que el punto de articulación de

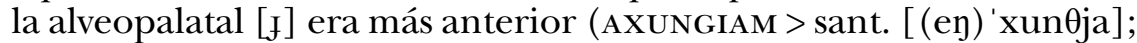

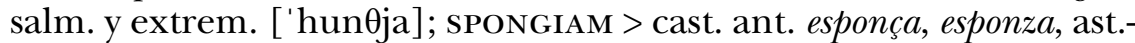
leon. espuncia, escuncia), y $\left[\mathrm{n}(\mathrm{t}) \int\right],[\mathrm{n}(\mathrm{d}) 3]$ fue el desenlace para aquellos otros hablantes o dialectos en los que el punto de articulación de la [J] era más posterior (sPONGIAM > gall. esponxa [es' ponfa]; port. [if'põza]; cat. [əs' pənzə] y cast. [es'poyxa]).

Por último, convendría detenerse en la posibilidad de que [nd] (enjundia, espundia, etc.) esté emparentada con -NGIV-. Como se acaba de comprobar más arriba, [nd] es la solución común para -NGIV-en francés (Pope 1934, § 293). No obstante, al utilizar [f] y [ [ ] mecanismos articulatorios antagónicos, aquí el cambio de la alveopalatal [f] en -NGIV- > [nJ] a la oclusiva alveolar [d] está crucialmente motivado por el contacto con la rótica del infinitivo una vez que la

12 Tanto en italorromance como en galorromance la preferencia por una u otra solución parece seguir criterios geográficos nítidos: [n3] es normal en italorromance septentrional, en lucano, pisano y toscano occidental, incluida Florencia, mientras que [n] es característica del toscano oriental y del italorromance meridional, salvo Sicilia y Calabria, donde se prefiere [ntf]; en galorromance la diferencia es sobre todo entre la mitad norte (francés) con [nd] y la mitad sur (occitano) con [n]. 
vocal postónica sincopa; no en vano, la mayoría de los ejemplos de -NGIV- > [nd] en francés corresponde a infinitivos sincopados. Con todo, -NGE,I- $>$ [nd] también ocurre sin entrar la alveopalatal en contacto con una rótica (cf. FRANGERE > ast. frander, frender; GINGIVAM > extrem. endiva). En estos casos, mucho más esporádicos que aquellos en los que hay una rótica involucrada, $[\mathrm{nJ}]>[\mathrm{nd}]$ puede deberse al incremento de la superficie de contacto entre el articulador activo y el pasivo. Dicho incremento está asociado a procesos de refuerzo articulatorio y puede traducirse en una extensión del contacto linguopalatal a regiones del paladar más anteriores. Ejemplos de esto son algunos dialectos galorromances, en los que [k] y [g] en [ka] y [ga] iniciales de palabra se pronuncian notablemente adelantadas (Rousselot 1924, p. 607), o el coreano, en donde [t] y [n] se articulan como dentoalveolares cuando se encuentran al comienzo de dominios prosódicos superiores (enunciado y frase entonativa), pero como alveolares cuando se encuentran al comienzo de dominios prosódicos inferiores (frase fonológica, palabra y sílaba) (Keating et al. 2003).

Puesto que la posición de inicio de sílaba tras consonante heterosilábica en interior de palabra es, junto con la de inicial de palabra, una de las asociadas con frecuencia a procesos de fortalecimiento (Ségéral y Scheer 2008), parece factible que la secuencia [nJ] salida de -NGIV- pasara a [nd] a través precisamente de un aumento de la magnitud del gesto de [J] en posición postnasálica. Datos procedentes de algunos dialectos del español peninsular contemporáneo en los que la alveopalatal fricativa $/ \mathrm{j} /$ se ha convertido en $[\mathrm{d}]$ en los contextos $/ \# \mathrm{j} \mathrm{V} / \mathrm{y} / \mathrm{Vn} . \mathrm{j} \mathrm{V} /$ respaldan esta conjetura: hierro $\rightarrow$ ['djero] (ALECant, mapa 993, hierro); yendo $\rightarrow$ ['djendo] (ALECant, mapa 1174, yendo); yesca $\rightarrow$ ['djeska] (ALECant, mapa 768, yesca), salm. diezca 'yesca' (DEEH, s.v. esca); inyección $\rightarrow$ [indi'Ojon] (ALECMAN, mapa FON-203, inyección).

Otra ventaja de plantear la evolución -NGIV- $>\left[n_{J}\right]>[$ nd $]$ es que permite justificar mejor la convivencia en diversas áreas de la Península Ibérica de formas con [nd] y con [n $\theta$ ] que tienen el mismo significado. En las provincias de Palencia, Burgos, Zamora, Salamanca, Ávila y Cáceres coexisten variantes de enjundia y esponja con [nd] ([(ey) 'xundja], ['hundja], [e $\mathrm{e}^{\mathrm{h}}$ pundja], etc.) y $\operatorname{con}[\mathrm{n} \theta]$ ([ (en)'xun $\theta j \mathrm{j}$ ],

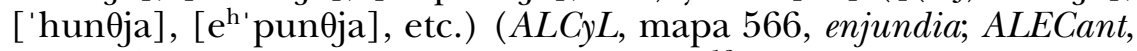
mapa 578, enjundia; Espinosa 1935, § 20) ${ }^{13}$. Ante esta situación, se

13 Algunos hechos interesantes que reflejan el ALECant y el $A L C y L$ acerca de la distribución de las variantes de enjundia es que en Santander no hay casos de [nd],

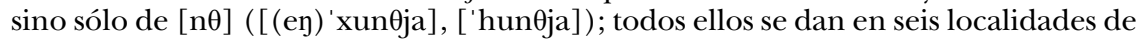
la mitad oriental de la provincia (Castro Urdiales, Penilla de Toranzo, Villacarriedo, Castillo Pedroso, Arredondo y Veguilla). En Castilla y León, la variante minoritaria en todas las provincias es la que tiene [n$\theta]$, salvo en Ávila, en donde es mayoría (trece casos de $[\mathrm{n} \theta]$ frente a cuatro de [nd]). Lo corriente en todos los lugares en los que [nd] y [n $\theta]$ alternan es su discontinuidad y su mutua superposición diatópica. 
podría pensar que -NGIV- $>\left[n_{J}\right]>[\mathrm{nd}]>[\mathrm{n} \theta]$, pero fonéticamente el cambio [nd] $>[\mathrm{n} \theta]$ se enfrenta a dos serios inconvenientes: la espirantización y el ensordecimiento de [d] tras nasal. Por el lado articulatorio, en posición postnasálica la constricción oclusiva en la cavidad oral necesaria para [d] es una prolongación de la configuración lingual creada para [n]; y por el lado aerodinámico, la liberación de aire a través del canal velofaríngeo requerida para la producción de la nasal favorece la vibración continuada de las cuerdas vocales durante [d] porque evita el incremento de la presión intraoral. Estos dos motivos hacen complicado sostener que la [d] deviniera contextualmente en una fricativa sorda tras nasal homorgánica, lo cual, en última instancia, no apoya que espuncia guarde filiación con espundia ${ }^{14}$. Por el contrario, estas dificultades para derivar $[n \theta]$ de [nd] sí prestan apoyo a la hipótesis de que el cambio -NGIV- $>$ [nJ] esté en la base tanto de $[\mathrm{n}]$ como de $[\mathrm{nts}] /[\mathrm{ndz}](>[\mathrm{n} \theta])$ y de [nd], lo que a su vez permite postular a AXUNGIAM y a SPONGIAM como los étimos de las variantes con estas soluciones en iberorromance.

Finalmente sobre este particular, un par de sugerentes derivados

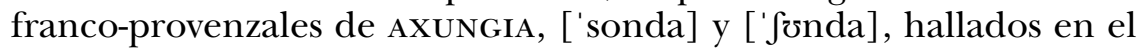
valle piamontés de Val Soana (cf. FEW, s.v. axungia), podrían utilizarse para atestiguar -NGIV- $>\left[n_{J}\right]>$ [nd] en romances no iberorrománicos en virtud de su similitud con el cast. enjundia, el port. enxúndia, etc. No obstante, parece más probable que se trate de casos de desafricación de una [dz] preexistente, ya que en otros valles piamonteses y aostanos aledaños los descendientes locales de AXUNGIA tienen afri-

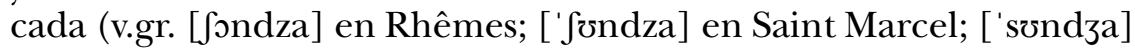
en Brussons; [ fundza] en Bruzolo) y en el norte de Italia la desafricación en [z] o [d] de la africada [dz] procedente de I- y (-) GE,I- latinas es un fenómeno relativamente generalizado (genovés ['ze:na] 'Génova'; veneciano rural ['ðugo] 'juego'; cf. Loporcaro 2011).

En conclusión, todos los datos presentados en esta sección avalan la idea de que AXUNGIAM y sPONGIAM, en cuanto que poseedoras de la secuencia -NGIV-, pueden ser los étimos de las variantes iberorrománicas de enjundia y esponja con [n], [nts], [ndz] $(>[\mathrm{n} \theta]),[\mathrm{n}(\mathrm{t})$ $\left.\int\right],\left[\mathrm{n}(\mathrm{d})_{3}\right]\left(>\left[\tilde{V}_{3}\right],[\mathrm{nx}]\right)$ y $[\mathrm{nd}]$.

14 Un aparente contraargumento a esto que se acaba de exponer es la pronunciación de los dientes como [lo ' $\theta$ jentə] en algunas localidades de Cáceres (Escurial) y Badajoz (Mirandilla, Helechosa de los Montes y Valencia de Mombuey) (GonzÁlez SAlgado 1999, mapa 361, diente). No obstante, la espirantización de la [d] en estos casos está influida por la aspiración de la [s] final de la palabra precedente (cf. nos vamos [no'famo] en el sur de España), como prueba el hecho de que en los lugares en que diente se recoge en singular lo hace siempre con oclusiva (['djentə]). 
Las secuencias -NGUL- han desembocado en dos posibles resultados en castellano: $[\mathrm{n}]$ o [K] (v.gr. CINGULUM > ceño, cello; SINGULARIUM $>$ señero; UNGULAM > uña) y [nd] (v.gr. COIUNGULAM > coyunda; SINGULOS $>$ sendos $)^{15}$. El primero de estos desarrollos conllevó la fusión articulatoria entre los gestos linguales correspondientes a [g] y [1] en el grupo [gl] tras la síncopa de la -U- átona, y la posterior asimilación progresiva o regresiva en $[\mathrm{n} K]\left(-\mathrm{NGUL}^{-}>[\mathrm{ngl}]>[\mathrm{ng} K]>[\mathrm{nJ} \mathrm{h}]>\right.$ $[\mathrm{n} \kappa]>[\mathrm{n}]$ o $[\kappa])$. El segundo desarrollo consistió en la asimilación progresiva en [ng] provocada por el enmascaramiento acústico de la nasal sobre oclusiva, seguido del fortalecimiento de la lateral postnasálica en [d] (-NGUL- $>[\mathrm{ngl}]>[\mathrm{y}(:) \mathrm{l}]>[\mathrm{nl}]>[\mathrm{nd}]$ ) (Gutiérrez 2018).

En coherencia con la hipótesis presentada en el apartado anterior, según la cual -NGIV- > [nd], se debe descartar que las variantes de enjundia y esponja que exhiben [nd] procedan de *AXUNGULAM y *spongulam. Aunque en castellano -NGUL- $>$ [nd] sí es posible, el intenso e irregular solapamiento entre las variantes con [nd] y las variantes con [n $\theta$ ] en las mismas áreas geográficas hace suponer que ambas se deben a evoluciones fonéticas diferentes a partir de un mismo étimo con -NGIV-, a saber, AXUNGIAM o SPONGIAM. En lo referente al gallego, aquí sólo consta un ejemplo con [nd]: espundia 'tumoraciones que le salen a las vacas en el vientre y la ubre' y 'verrugas' en el municipio orensano de Gudiña (cf. TLPGP). La evolución regular de -NGUL- en gallego es a [nK], no a [nd], por lo que se puede sospechar que este solitario espundia hallado, además, en una de las comarcas más orientales de Galicia es una importación de alguno de los iberorromances situados más al este.

Dejando, pues, [nd] de lado, el resto de evoluciones de -NGUL$([\mathrm{n} K]$ y $[K])$ encaja con los dos tipos de variantes de enjundia y esponja predominantes en el occidente peninsular (port. ant. enxunlha; port. enxulha; gall. enxunlla, enxulla, ensulla). Tal coincidencia entre esas soluciones de enjundia y esponja con $[\mathrm{n} K]$ o [K] en gallego y portugués con la zona donde precisamente las secuencias -NGUL- de otras voces latinas suelen desarrollarse en $[\mathrm{n} K]$ o $[K]-\mathrm{v} . g r$. *ANGULAREM $>$ gall. anllar, allar, GINGULUM > gall. cenllo, SINGULARIUM $>$ port. ant. senlheiro; SINGULOS > gall. ant. senllos, port. selhos; UNGULAM > gall. unlla (cf. DEEH; García de Diego 1978, p. 70; Williams 1962, $\S 92_{6}$ )- no puede ser casual, sino que responde seguramente a que estas variantes proceden de *AXungulam y *sPONGULAM.

Ante esta concurrencia territorial, la asunción de Corominas (DCECH, s.vv. chuleta y esponja) de que todas las variantes de enjundia y esponja en iberorromance son descendientes de AXUNGIAM y

15 También [t $\mathrm{t}$ ] , como en cingulum $>$ cincho. 
SPONGIAM pierde fuerza: razonar enxulha como una disimilación entre las nasales [n] y [n] de un hipotético * enxunya, y espulha como consecuencia de una analogía de esponya con unlha parece un tanto ad hoc dadas las circunstancias. Es cierto que existen ejemplos de intercambios entre [n] y [ $K$ ] que justificarían enxunya $>$ enxulha $-\mathrm{v}$.gr. $[\mathrm{n}]>[\Lambda]$ : SEMINIA > semilla, Guillermo $>$ Guiñermo; $[\kappa]>[\mathrm{n}]$ : DIABOLUM $>$ sant. diaño (García Lomas 1966), cat. dianya (cf. DCVB, s.v. diable)-, pero estos intercambios no explican la escisión de una consonante como [n] en una secuencia de dos consonantes como [nK] en estas formas gallegas y portuguesas.

Igual de problemática, pero en el sentido contrario, es la pretensión de García de Diego (DEEH, s.vv. *axungula y *spongula) de que *axungulam y *spongulam sean la fuente de las formas con [n $\theta$ ], [nx] y [nt]] (véanse supra $3 b$ y $5 b$ ). En primer lugar, este autor sitúa [n $\theta]$ como producto tanto de SPONGIAM (la africada alveolar del cast. ant. esponza hubiera evolucionado a la fricativa interdental) como de *spongulam (leon. espuncia; extrem. espuncia). La causa podría estar en que García de Diego hubiera aceptado la propuesta de Espinosa $(1935, \S 20)$, conforme a la cual la terminación -úncia en juncia y espuncia se debe a una analogía sobre enjundia y espundia para acomodar sus terminaciones -úndia a un patrón fonético más habitual en castellano (pronuncio, renuncio, anuncio). De ser así, estas variantes salmantinas y extremeñas con [n $\theta]$-a las que se podrían sumar las de Santander, Palencia, Zamora, Burgos, Ávila y Segovia recogidas en el $A L C y L$ y en el ALECant-vendrían de [nd]. De todos modos, Espinosa parte en su estudio de AXUNGIAM y SPONGIAM, cuyas secuencias -NGIV-, al igual que las -NTIV- de PRONUNTIO > pronuncio, RENUNTIO $>$ renuncio y ANUNTIO > anuncio, son evolucionables a [n $\theta]$. Por esta razón, y por las complicaciones fonéticas ya señaladas en el apartado anterior que entraña el cambio [nd] $>[n \theta]$, es más aconsejable no recurrir a [nd] como estadio intermedio entre -NGIV-y [n $\theta]$, sino traer $[\mathrm{n} \theta]$ directamente de -NGIV-.

Otro de los resultados vinculados por García de Diego a *SPONGULAM es, en segundo lugar, [yx], que implica el estadio previo [n3] (y $\left.\left[\mathrm{n} \int\right]\right)$. A falta de más detalles, es presumible que García de Diego, considerando el comportamiento de los grupos secundarios [gl] intervocálicos (v.gr. COAGULUM > cuajo; REGULAM > reja), derivara [n3] de $[\mathrm{n} K](-\mathrm{NGUL}->[\mathrm{ngl}]>[\mathrm{ng} K]>[\mathrm{nf} K]>[\mathrm{n} K]>[\mathrm{n} 3])$. Sin embargo, no hay evidencias, más allá del propio caso de esponja, de que los grupos [gl] de las secuencias [ngl] se desarrollaran en castellano hacia la fricativa [3]; por el contrario, estos grupos van a parar normalmente a $[n]$ y con menos frecuencia a $[K]$ y [nd]. Siendo esto así, y en vista de la abundancia de $[\mathrm{n}(\mathrm{t}) \mathrm{f}]$ y $\left[\mathrm{n}(\mathrm{d})_{3}\right]$ a partir de -NGIV-en gallego, aragonés y catalán, parece recomendable situar el cast. esponja en la órbita de spongiam, en vez de en la de *sPongulam. 
En este sentido, otro argumento complementario para defender que SPONGIAM es el étimo del castellano esponja lo tenemos en el área gallego-portuguesa. En esta zona, que es en donde con más intensidad se manifiestan las soluciones $[\mathrm{n} K]$ y $[K]$ a partir de -NGUL-, se encuentran desde el siglo Xv formas con grafías que denotan una [3] -gall. esponsa (cf. TMILG); port. sponia (cf. Cunha 1994)- y cuyo significado no es el de 'verruga, tumor o úlcera de las ganaderías', sino el de 'esponja', es decir, 'utensilio para absorber líquidos'. Estas formas, con este significado, son los antecedentes claros de las actuales esponxa [es'ponfa] en gallego (con ensordecimiento de la sonora), y esponja [if'põza] en portugués. Estas evoluciones se corresponden con las experimentadas por otras palabras con la secuencia [ $(\mathrm{y}) \mathrm{gj}$ V] en los iberorromances occidentales -v.gr. ANGELUM > gall. anjo, anxo ['anfo]; PULEGIUM > gall. poenjo, poenxo, poexo [po'efo]; RIGIDUM > gall. rejo, rexo ['refo] (DPLG; Mariño Paz 2017, § 2.2.1.1.3); ANGELUM > port. anjo ['ãzu]; FUGIO > port. fujo ['fuzu]; RIGIDUM > port. rijo [' вizu] (Williams 1962, $\S 89_{7}$ ). Tanto esta correspondencia fonética como la marcada disparidad entre los significados de las soluciones con [3]/ $\left[\int\right]$ y los de las soluciones con [n $\left.\Lambda\right]$ y [ $\left.K\right]$ muestran suficientemente que el gall. esponxay el port. esponja tienen que estar relacionados con SPONGIAM y no con *SPONGULAM.

Para terminar, [nt $]$, atestiguado en el ribagorzano esponchar. En los iberorromances orientales (aragonés y catalán), los grupos [kl] y [gl] tras consonante se mantienen inalterables (Zamora Vicente 1967, p. 248). Una idiosincrasia del ribagorzano es que estos grupos llegan a palatalizar (v.gr. cINGULAM > singlla; cf. Haensch 1961-62), pero no avanzan más allá. Por esta razón, podría ser más adecuado admitir que esponchar procede de SPONGIAM, sobre todo si se tiene en cuenta que su cognado en valenciano -variedad situada también en la Iberorromania oriental- es pronunciado con la africada alveopalatal sorda ([es'pontfa]) (cf. DCVB, s.v. esponja).

En resumen, -NGUL- es la fuente de $[n K]$ y $[K]$, que son los resultados predominantes en el occidente de la Península.

\section{ANÁlisis MORFOLÓGICO}

Una vertiente aún no examinada en este artículo es la morfológica: los diminutivos derivados de las bases latinas AXUNGIA y SPONGIA deberían ser AXUNGIOLA y SPONGIOLA. Las evidencias para afirmar esto radican tanto en el tratamiento de otras bases similares en latín (filius $\rightarrow$ filiolus, linteum $\rightarrow$ linteolum, malleus $\rightarrow$ malleolus) como en la atestiguación directa de los susodichos diminutivos. Goetz (CGL, t. 3, pp. 590, 595, 611, 623 y 629), de quien se hace eco el TLL (s.v. axungiola), documenta axungiola en varios glosarios griego-latinos 
(Hermeneumata codicis Vaticani Reginae Christinae 1260, siglo x; Hermeneumata Bernensia, siglo xI; Codex Vaticanus 4417, siglo x u XI). En realidad, las formas que aparecen en estas obras son exungiola y sungiola, a las cuales Goetz (CGL, t. 7, p. 257) interpreta como variantes de axungiola. Sin embargo, el significado 'grasa animal' de axungia lleva a André (1956) a plantear que no nos encontramos ante variantes de axungiola, sino ante variantes de spongiola: semánticamente, spongea y spongiola se usan desde Plinio (Naturalis historia, lib. 29, 94 y lib. 25, 18) para referirse a la agalla esponjosa formada en un escaramujo por la picadura de un mosquito y cuya excrecencia espumosa tenía fines medicinales en la antigüedad; fonéticamente, tras la prótesis de [e] en la secuencia consonántica inicial de spongiola, [s] y [p] metatetizaron pasando después la oclusiva labial a velar-André da como ejemplos adicionales de este proceso psimithium > eximitium y diapsimithium > diaximicium. La similitud formal entre exungiola y un posible diminutivo de axungia hizo que esta confusión se perpetuase.

Por tanto, si es patente que AXUNGIOLA y sPongiola son los diminutivos de AXUNGIA y SPONGIA, ¿por qué se recurre en este artículo a las formas reconstruidas *AXUNGULAM y *SPONGULAM? La respuesta es por razones fonéticas y dialectales. Como se ha intentado demostrar en las páginas precedentes, las -NGIV- de AXUNGIAM y SPONGIAM no parecen ser la fuente ni de $[\mathrm{n} \kappa]$ ni de $[\kappa]$. Ello se puede concluir de los siguientes hechos: 1) la palatalización de [gj] no genera de primeras laterales palatales en ninguna lengua románica, sino un abanico de fricativas o africadas alveolares o alveopalatales; 2) los recursos de Corominas a la disimilación entre las nasales [n] y [n] en *ensunya $>$ ensulha y a la analogía de unlha sobre esponya son excesivamente arbitrarios. Mucho menos pueden haberse originado [n $\kappa]$ y $[K]$ de las -NGIV- de AXUNGIOLA y SPONGIOLA, en las que la vocal tónica hubiera evitado el contacto entre [g] y [1], y que en gallego y portugués hubieran elidido la [1] intervocálica ${ }^{16}$.

En oposición a esto, creo que la clave para determinar la procedencia de las variantes de enjundia y esponja con $[\mathrm{n} K]$ y $[K]$ en iberorromance está en que la ubicación geográfica de todas estas variantes coincide plenamente con el occidente de la Península Ibérica, que es el área en que las secuencias -NGUL- de otras palabras latinas han evolucionado con regularidad a [nK] y [K] (cf. García de Diego 1978, p. 70, y Williams 1962, $\S 92_{6}$ ). Siendo esto así, atribuir a una mera casualidad esta coincidencia se antoja un argumento tan oportunista y lábil que es muy difícil, en mi opinión, no desconfiar de él y rechazarlo.

Es verdad que el riesgo de proponer *AXUNGULAM y *SPONGULAM reside en la creación de dos nuevos étimos no atestiguados. Este

16 A causa de la insólita apócope de [o] en portugués, Williams (1962, § 48 7 ) piensa que los resultados en esta lengua de tal tipo de diminutivos latinos (ANELLUM $>$ port. anel, LINTEOLUM $>$ lençol) son en realidad galicismos. 
proceder se puede considerar tan ad hoc como otros, ya que sin el debido cuidado puede dar pie a la creación casi a placer de tantas formas reconstruidas como sean necesarias para dar solución a todas aquellas voces romances con etimologías difíciles. Llevado al extremo, puede llegar a constituir un problema metodológico muy serio en la labor etimológica; pero lo contrario también: tan peligroso es acuñar indiscriminadamente étimos reconstruidos como no hacerlo nunca; todo depende de los argumentos que se aduzcan para ello. En este caso, aunque no haya evidencia ni directa ni indirecta de AXUNGA y SPONGA en latín, y aunque, por consiguiente, los orígenes de *axungulam y *spongulam sean nebulosos, me parece que el análisis fonético y los datos dialectales romances capacitan razonablemente para postular estos diminutivos reconstruidos ${ }^{17}$.

\section{Conclusión}

En la investigación llevada a cabo en este trabajo se ha ofrecido una explicación fonéticamente motivada sobre la evolución de las secuencias latinas -NGIV-y -NGUL- en iberorromance. Dicha explicación ha mostrado la necesidad de matizar o corregir las propuestas existentes sobre la etimología de las variantes de enjundia y esponja en este dominio lingüístico. Según las conclusiones alcanzadas, enjundia, esponja y sus respectivas variantes en la Península Ibérica se pueden agrupar en torno a AXUNGIAM/ SPONGIAM y *AXUNGULAM/*SPONGULAM de la manera siguiente:

1. En gallego y en portugués, las variantes con $[\mathrm{n} \kappa]$ y $[K]$ derivan de *AXungulam y *spongulam, mientras que aquellas con [n(d)3], $\left[\mathrm{n}(\mathrm{t}) \int\right]$ o $\left[\tilde{V}_{3}\right]$ derivan de AXUNGIAM y SPONGIAM.

2. Las variantes del resto de iberorromances descienden de AXUNGIAM y SPONGIAM y, de manera más concreta, del estadio inmediatamente posterior a -NGIV-, es decir, [nj]:

a) $[\mathrm{n}]$ : se localiza en los iberorromances orientales (aragonés y catalán).

17 Vale la pena recordar ahora unas palabras muy pertinentes de GARCía DE Diego (1926, p. 20): "Es evidente que con sola la fonética no pueden hacerse etimologías, pero no lo es menos que sin fonética el etimologista carece de un necesario instrumental. Si de un lado hay que restringir el método fonético, no aplicándolo más que a las voces que siguieron su ruta, de otro hay que perfeccionarlo y complicarlo; porque el hecho fonético no es siempre tan uniforme como nuestras primeras leyes lo suponían". Y otras de Michelena (1990, p. 52): "no debe olvidarse que en la reconstrucción el primer paso, el que se fundamenta en la admisión de cambios fónicos regulares, es no sólo el primero y el que posibilita la mayor parte de los demás, sino que además es el que se asienta en un terreno verdaderamente firme. No hay razón para descuidar esta jerarquía”. 
b) $[\mathrm{nts}],[\mathrm{ndz}]>[\mathrm{n} \theta]$ : a grandes rasgos, se extienden por las provincias de Santander, Burgos, Palencia, Zamora, Salamanca, Ávila, Segovia y Cáceres; también se conoce un par de ejemplos en sendos textos castellanos de los siglos XIII y XV.

c) $\left[\mathrm{n}(\mathrm{t}) \int\right],\left[\mathrm{n}(\mathrm{d})_{3}\right],\left[\tilde{\mathrm{V}}_{3}\right]$ y $[\mathrm{yx}]$ : se hallan en portugués, gallego, castellano y en catalán.

d) [nd]: la mayor densidad de variantes con este resultado (enjundia, espundia) se da en el centro peninsular, y disminuye a medida que nos desplazamos hacia el este o hacia el oeste.

Además del propósito específico de establecer la filiación etimológica de esta serie de voces iberorromances, otro propósito más general de esta investigación ha sido el de enfatizar la idea de que disponer de un conocimiento más refinado y detallado de la fonética histórica de las lenguas iberorrománicas puede contribuir decisivamente a los estudios en etimología e historia del léxico de esta subfamilia.

\section{REFERENCIAS}

\section{Corpus}

CORDE $=$ Real Academia Española: Banco de datos (CORDE) [en línea]. Corpus diacrónico del español, http://www.rae.es [consultado en enero de 2018].

$C G L=$ Gustav Loewe et Georg Goetz 1889-1923. Corpus glossariorum latinorum, B.G. Teubner, Leipzig, 7 ts.

\section{Atlas lingüisticos}

ALCyL = Manuel Alvar 1999. Atlas lingüístico de Castilla y León, Junta de Castilla y LeónConsejería de Educación y Cultura, Salamanca, 3 ts.

ALECant = Manuel Alvar 1995. Atlas lingüístico y etnográfico de Cantabria, Arco/Libros, Madrid.

ALECMAN = Pilar García Mouton y Francisco Moreno Fernández 2003. Atlas lingüistico (y etnográfico) de Castilla-La Mancha, http:/ / www.linguas.net/alecman/ [consultado en enero de 2018].

\section{Repertorios lexicográficos}

Cunha, Antônio Geraldo da 1994. Dicionário etimológico Nova Fronteira da língua portuguesa, Nova Fronteira, Rio de Janeiro.

$D A=$ Asociación de Academias de la Lengua Española (ASALE) 2010. Diccionario de americanismos, Santillana, Madrid.

DCECH = Joan Corominas y José Antonio Pascual 1980-1991. Diccionario crítico etimológico castellano e hispánico, Gredos, Madrid, 6 ts. 
DCELC = Corominas, Joan 1955-1957. Diccionario crítico etimológico de la lengua castellana, Gredos-A. Francke A.G., Madrid-Bern, 4 ts.

$D C V B=$ Antoni Maria Alcover y Francesc de Borja Moll 1930-1962. Diccionari catalàvalencià-balear, Editorial Moll, Palma.

DEEH = Vicente García de Diego 1985. Diccionario etimológico español e hispánico, Espasa-Calpe, Madrid.

DPLG = Xosé Luís Regueira (dir.) 2010. Dicionario de pronuncia da lingua galega, Instituto da Lingua Galega, Santiago de Compostela.

Ferraz y Castán, Vicente 1934. Vocabulario del dialecto que se habla en la Alta Ribagor$z a$, Tipografía de Archivos, Madrid.

FEW = Walther von Wartburg 1922-2002. Französisches Etymologisches Wörterbuch, Zbinden, Basel.

Neira Martínez, Jesús y M. Del Rosario Piñeiro 1989. Diccionario de los bables de Asturias, Instituto de Estudios Asturianos, Oviedo.

$R E W=$ Wilhelm Meyer-Lübke 1930-1935. Romanisches Etymologisches Wörterbuch, Winter, Heidelberg.

$T L L=$ Thesaurus linguae latinae 1900-. Deutsche Akademie der Wissenchaften, Berlin.

TLPGP = Rosario Álvarez (coord.). Tesouro do léxico patrimonial galego e portugués, Instituto da Lingua Galega, Santiago de Compostela, http://ilg.usc.es/Tesouro [consultado en enero de 2018].

TMILG = Xavier Varela Barreiro (dir.) 2004. Tesouro Medieval Informatizado da Lingua Galega, Instituto da Lingua Galega, Santiago de Compostela, http://ilg.usc.es/ tmilg [consultado en febrero de 2018].

\section{Referencias bibliográficas}

Alves Pereira, F. 1933. "Glossario dialectologico do concelho dos Arcos de Valdevêz”, Revista Lusitana, 31, pp. 292-300.

Andolz, Rafael 1991. Diccionario aragonés, $5^{\text {a }}$ ed., Mira, Zaragoza.

ANDRÉ, Jacques 1956. "Contribution à l'explication des gloses de médecine”, Bulletin du Cange. Archivum Latinitatis Medii Aevi, 26, 3, pp. 185-211.

Ariza, Manuel 2012. Fonología y fonética históricas del español, Arco/Libros, Madrid.

Badía Margarit, Antonio 1951. Gramática histórica catalana, Noguer, Barcelona.

Casado lobato, María Concepción 1948. El habla de la Cabrera Alta. Contribución al estudio del dialecto leonés, CSIC, Madrid. (Anejos de la Revista de Filología Espanola, 44).

Castro, Américo 1921. "Unos aranceles de aduanas del siglo XiII", Revista de Filología Española, 8, pp. 325-356.

Cornu, Jules 1906. "Die portugiesische Sprache", en Grundriss der romanischen Philologie. Hrsg. Gustav Gröber, Karl J. Trübner, Strassburg, t. 1, pp. 916-1037.

Espinosa, Aurelio M. 1935. Arcaísmos dialectales. La conservación de " $s$ ” " $z$ ” sonoras en Cáceres y Salamanca, Casa Editorial Hernando, Madrid.

García de Diego, Vicente 1926. Problemas etimológicos. Discurso leído ante la Real Academia Española en el acto de su recepción por D. Vicente García de Diego, Tipografía y encuadernación de Senén Martín, Ávila.

García de Diego, Vicente 1943. Contribución al diccionario hispánico etimológico, CSIC, Madrid. (Anejos de la Revista de Filología Española, 2).

García de Diego, Vicente 1978. Manual de dialectología española, Centro Iberoamericano de Cooperación, Madrid.

García lomas, Adriano 1966. El lenguaje popular de la Cantabria montañesa, Aldus, Santander. 
González Salgado, José Antonio 1999. Cartografia lingüistica de Extremadura. Origen y distribución del léxico extremeño, tesis, Universidad Complutense de Madrid, Madrid.

Gutiérrez, CÉsar 2018. "Competencia entre palatalización y nasalización en las secuencias -NGUL- en castellano”, Zeitschrift für Romanische Philologie, 134, pp. 404-418.

Haensch, GÜNTher 1961-62. "Las hablas de la Alta Ribagorza (Pirineo aragonés). (Continuación)", Archivo de Filología Aragonesa, 12/13, pp. 117-250.

Keating, Patricia, Cho Taehong, Cécile Fougeron \& Chai-Shune Hsu 2003. "Domain-initial articulatory strengthening in four languages", en Phonetic interpretation: Papers in laboratory phonology VI. Eds. John Local, Richard Ogden, Rosalind Temple, Mary E. Beckman \& John Kingston, Cambridge University Press, Cambridge, pp. 145-163.

Lapesa, Rafael 1981. Historia de la lengua española, Gredos, Madrid.

Lloyd, Paul M. 1993. Del latín al español. T. 1: Fonología y morfología históricas de la lengua española, Gredos, Madrid.

Loporcaro, Michele 2011. "Phonological processes", en The Cambridge History of the Romance Languages. Eds. Martin Maiden, John Charles Smith \& Adam Ledgeway, Cambridge University Press, Cambridge, t. 1, pp. 109-154.

Lourenço Loução, JoÃo Luis 1931. "Lexicografia das margens do Minho", Revista Lusitana, 29, pp. 246-276.

Mariño Paz, RAMÓN 2017. Fonética e fonoloxía históricas da lingua galega, Edicións Xeraisde Galicia, Vigo.

Menéndez Pidal, Ramón 1941. Manual de gramática histórica española, Espasa-Calpe, Madrid.

Meyer-Lübke, Wilhelm 1890. Grammaire des langues romanes, t. 1, H. Welter, Paris.

Michaëlis de Vasconsellos, Carolina 1910. "Mestre Giraldo e os seus tratados de Alveitaria e Cetraria”, Revista Lusitana, 13, pp. 149-432.

Michelena, Luis 1990 [1963]. Lenguas y protolenguas, Diputación Foral de Guipúzcoa, San Sebastián. (Anejos del Anuario del Seminario de Filología Vasca "Julio de Urquijo", 20. Serie Obras completas de Luis Michelena, 2).

Moll, FranCESC DE BorJa 1952. Gramática histórica catalana, Gredos, Madrid.

Penny, Ralph 2006. Gramática histórica del español, Ariel, Barcelona.

Pereira, Gabriel 1909. "Libro d'alveitaria do Mestre Giraldo", Revista Lusitana, 12, pp. 1-60.

Pope, Mildred K. 1934. From Latin to Modern French with especial consideration of AngloNorman, Manchester University Press, Manchester.

Recasens, DANiEl 2011. "Velar and dental consonant softening in Romance", Diachronica, 28, 2, pp. 186-224, doi: 10.1075/dia.28.2.02rec.

Recasens, Daniel \& Aina Espinosa 2006. "Articulatory, positional and contextual characteristics of palatal consonants: Evidence from Majorcan Catalan", Journal of Phonetics, 34, pp. 295-318, doi:10.1016/j.wocn.2005.06.003.

Recasens, DANiel \& Aina Espinosa 2009. "Acoustics and perception of velar softening for unaspirated stops", Journal of Phonetics, 37, pp. 189-211, doi:10.1016/j. wocn.2009.01.0011.

ROHLFS, GERHARD 1966. Grammatica storica della lingua italiana e dei suoi dialetti. Fonetica, Giulio Einaudi, Torino.

Rousselot, L'abbé Pierre-Jean 1924. Principes de phonétique expérimentale, H. Didier, Paris.

Ségéral, Philippe \& Tobias Scheer 2008. "Positional factors in lenition and fortition”, en Lenition and fortition. Eds. Joaquim Brandão de Carvalho, Tobias Scheer \& Philippe Ségéral, Mouton de Gruyter, Berlin, pp. 131-172.

Tallgren, Oiva Johannes 1907. Estudios sobre la Gaya de Segovia, Kirjapaino-Osakeyhtiö Sana, Helsinki. 
Williams, Edwin B. 1962. From Latin to Portuguese. Historical phonology and morphology of the Portuguese languages, University of Pennsylvania Press, Philadelphia.

Zamora Vicente, Alonso 1967. Dialectología española, Gredos, Madrid.

ZAMPAUlo, ANDRÉ 2013. "Los caminos diacrónicos de la lateral palatal en la historia del castellano: evidencia cronológica, comparativa y dialectal para una nueva propuesta”, Revista de Historia de la Lengua Española, 8, pp. 149-174. 